\title{
Desigualdades de género relacionadas con la flexibilidad laboral
}

\section{Gender inequalities related to labor flexibility}

\section{Imma Cortès-Franch ${ }^{1,2,3}$}

Lucía Artazcoz ${ }^{1,2,3,4}$

1Agència de Salut Pública de Barcelona, Barcelona, España.

${ }^{2}$ CIBER en Epidemiología y Salud Pública (CIBERESP), Madrid, España.

${ }^{3}$ Institut de Recerca Biomèdica (IIB-Sant Pau), Barcelona, España.

${ }^{4}$ Centre d'Investigació en Salut Laboral (CiSAL), Universitat Pompeu Fabra, Barcelona, España.

Fechas · Dates

Recibido: 2021.09.15

Aceptado: 2021.10.08

Publicado: 2021.10.15
Correspondencia · Corresponding Author

Imma Cortés

icortes@aspb.cat 


\section{La flexibilidad laboral}

Desde mediados de la década de 1970 se han producido profundos cambios en las economías capitalistas occidentales relacionados con factores tecnológicos, políticos y económicos, entre los que destacan la globalización, la liberalización de las políticas laborales, el debilitamiento sindical y las nuevas tecnologías. Estos cambios han ido acompañados de un aumento progresivo de la flexibilidad laboral impulsada por necesidades de las empresas, incrementada en periodos de crisis económica como la de mediados de los años 1990 y la de 2008.

Las estrategias de flexibilidad laboral son diversas. Pueden referirse a las jornadas laborales (tanto en el número de horas trabajadas como en la organización de la jornada), a los salarios, la subcontratación de personas o empresas, y una de las más importantes: la variación del número de personas empleadas mediante la contratación temporal, el despido, etc. En cualquier caso, el aumento de la flexibilidad ha dado lugar a un incremento de nuevas formas de ocupación caracterizadas por altos niveles de inestabilidad laboral y una erosión general de las condiciones de trabajo y ocupación de los trabajadores y trabajadoras. Son formas de ocupación que se acumulan en los grupos de personas en una situación de más vulnerabilidad en el mercado de trabajo debido a su falta de poder. En términos generales, y siguiendo los ejes de desigualdad social, son las personas jóvenes, las personas migradas, las mujeres y las personas con menos nivel de estudios ${ }^{(1)}$.

La investigación sobre el impacto en la salud y el bienestar de las condiciones de trabajo y ocupación relacionadas con la flexibilidad laboral demuestra, en general, consecuencias negativas para la salud y el bienestar, así como para las perspectivas laborales, principalmente por la mayor inestabilidad y las condiciones laborales más deficientes ${ }^{(2)}$. Se han constatado impactos negativos en la satisfacción laboral y con la vida, en la salud percibida y sobre todo en la salud mental (trastornos psicológicos, distrés mental y depresión) ${ }^{(3)}$. Aun así, los resultados son heterogéneos, con algunas dimensiones con una sólida evidencia del impacto negativo, como el paro y la inseguridad laboral y, en cambio, otras con resultados menos concluyentes como la temporalidad o todavía incipientes, como la precariedad o la calidad del trabajo. En cualquier caso, hay que destacar la escasa investigación sobre las desigualdades sociales en este ámbito y especialmente las desigualdades de género.

\section{Las desigualdades de género en salud relacionadas con la flexibilidad laboral}

Una aproximación a las desigualdades de género en salud relacionadas con la flexibilidad laboral requiere tener en cuenta, al menos, los siguientes aspectos:

\section{Falta de investigación}

Se ha documentado una mayor prevalencia de condiciones ligadas a la flexibilidad laboral en las mujeres, por ejemplo, las mujeres trabajan más sin contrato, con 
contratos temporales de duración determinada y también tienen más inseguridad labora( ${ }^{(4)}$. Igualmente, las tasas de paro suelen ser más elevadas en ellas, los salarios más bajos y trabajan más en jornadas parciales. A pesar de esta situación más desfavorable, hay muy poca investigación sobre las desigualdades de género en salud relacionadas con la flexibilidad. Además, es frecuente que los estudios no analicen las posibles asociaciones de la flexibilidad con la salud y el bienestar de manera separada en hombres y mujeres, sino que traten el sexo como variable de ajuste. Por ejemplo, en una revisión sobre flexibilidad laboral con 10 estudios longitudinales, solo uno separó el análisis por sexo(5). 0 todavía más, que solo incluyan muestras de hombres ${ }^{(6,7)}$.

\section{La división de género del trabajo}

El trabajo remunerado y el trabajo no remunerado del entorno familiar están estrechamente relacionados en las mujeres. En la mayor parte de países la responsabilidad del trabajo doméstico y familiar recae en las mujeres, lo cual determina su menor participación en el mercado laboral, el menor poder en el puesto de trabajo y la división horizontal de género, es decir, la sobrerrepresentación en puestos de trabajo que en buena medida reproducen el rol del trabajo doméstico y familiar y que son infravalorados en relación con los trabajos más masculiniza$\operatorname{dos}^{(8)}$. Esta división de género tiene como consecuencia diferencias sustanciales en las condiciones de trabajo y ocupación relacionadas con la flexibilidad laboral, la sobrecarga y dificultades de compaginación del trabajo remunerado y el trabajo no remunerado doméstico y de cuidado de personas dependientes, además de la exposición a riesgos laborales específicos de las ocupaciones feminizadas, como por ejemplo las de cuidados.

\section{La importancia de los contextos}

La incipiente investigación sobre el rol del contexto socioeconómico y político sobre las desigualdades en salud relacionadas con la flexibilidad laboral va sumando evidencia respecto al rol de las diferentes tipologías de estados del bienestar, que a la vez están estrechamente relacionadas con las políticas que regulan los mercados laborales y las políticas familiares. Por ejemplo, en los países del Norte de Europa no se han encontrado diferencias entre el estado de salud de los trabajadores precarios y sus homólogos en puestos de trabajo permanentes ${ }^{(9)}$. Tampoco se han encontrado diferencias en la salud mental entre las personas que ocupan puestos de trabajo de buena calidad, puestos de baja calidad y las que están desempleadas. Estos resultados se han relacionado con las generosas prestaciones de desempleo, el elevado gasto en políticas activas de ocupación, las leyes de protección del despido y las normas laborales sólidas y completas, características de estos países. Tampoco se han encontrado diferencias entre hombres y mujeres en esta relación ${ }^{(10)}$. En este sentido, los países del norte de Europa, que suelen tener mejores condiciones de equidad de género que el resto de los países europeos, tienen políticas familiares que apoyan el modelo de doble proveedor, mientras que la atención a personas dependientes se basa en la prestación de servicios públi- 
cos, y consecuentemente, las mujeres pueden tienen una mayor participación en el mercado laboral ${ }^{(11)}$.

El contexto familiar también es un determinante de las desigualdades de género en salud relacionadas con la flexibilidad laboral. Aunque es una cuestión muy poco analizada, algunos estudios apuntan que en países con modelos tradicionales de familia (los hombres con el papel de sustentadores económicos principales y las mujeres responsables del trabajo doméstico y de cuidados), como por ejemplo los países del sur de Europa, el impacto de la inestabilidad laboral en la salud mental es superior en los hombres casados e inexistente en las mujeres casadas, pero hay pocas diferencias entre hombres y mujeres separados, divorciados y solte$\operatorname{ros}^{(12)}$.

\section{El impacto desigual de las crisis}

En un momento en que las consecuencias en el ámbito laboral de la actual crisis derivada de la pandemia de COVID-19 ya son graves y se espera que se incrementen en el corto y medio plazo, es pertinente una reflexión sobre el posible impacto en las desigualdades de género en salud y bienestar relacionadas con la flexibilidad laboral.

Sabemos de las anteriores crisis económicas que las mejoras logradas en las condiciones de trabajo y ocupación corren el riesgo de perderse y que las desigualdades de género existentes se agravan todavía más. En un reciente documento de la Organización Internacional del Trabajo se identificaban 5 lecciones aprendidas de crisis anteriores respecto a las desigualdades de género relacionadas con la flexibilidad laboral (13):

El género es un mecanismo de restricción cuando los trabajos son escasos. En la primera
fase de la crisis económica de 2008 hubo un rápido y enorme incremento del paro en los
hombres, sobre todo en los países del sur de Europa, debido a la mayor presencia mascu-
lina en sectores como la construcción y las industrias relacionadas. Pero la recuperación
de la ocupación femenina después de la crisis fue mucho más lenta. De hecho, cuando los
trabajos no son suficientes se tiende a negar a las mujeres las oportunidades laborales y la
seguridad respecto a los hombres. Hay que tener en cuenta que, a diferencia de otras crisis,
el impacto laboral de la COVID-19 está siendo muy relevante en sectores feminizados como
el trabajo doméstico, el comercio no esencial o el sector turístico.

La flexibilidad laboral y algunas políticas que la favorecen como la desregulación de los mercados de trabajo, conducen a la expansión de la ocupación precaria de las mujeres. La progresiva desregulación se ha incrementado en épocas de crisis con el objetivo de aumentar el número de puestos de trabajo, pero ha tenido como consecuencia la disminución de la calidad de estos. Las situaciones de vulnerabilidad que llevan a aceptar malas condiciones de trabajo y ocupación son determinantes de efectos negativos en la salud y el bienestar. En la crisis de 2008, para compensar la pérdida de trabajo de sus parejas, muchas mujeres entraron al 
mercado laboral o incrementaron el número de horas, teniendo que aceptar condiciones precarias para mantener el trabajo, lo cual se asoció a una peor salud ${ }^{(14)}$.

El trabajo doméstico y de cuidado familiar se incrementa cuando las mujeres se quedan en paro, a diferencia de los hombres. El paro se ha asociado a un menor impacto en la salud de las mujeres en comparación con los hombres en países con modelos de familia tradicional por la oportunidad que tienen ellas de desarrollar el rol alternativo de cuidados cuando se pierde el trabajo remunerado. Aun así, algunos estudios sugieren que este efecto se restringiría a las mujeres casadas, y que en momentos de crisis económica en países que tienen también una elevada precariedad laboral, como los del sur de Europa, el efecto desaparecería ${ }^{(15)}$.

Las mujeres se ven afectadas directa e indirectamente cuando se llevan a cabo recortes en el gasto público. Por un lado, se pierden puestos de trabajo y empeoran las condiciones (incluidos los salarios) de sectores muy feminizados como la administración pública y los sectores asistenciales. Por otro lado, las mujeres aumentan su dedicación a las tareas de cuidado familiar por la disminución de las prestaciones de los servicios públicos. En este sentido, en las crisis económicas, el trabajo doméstico y de cuidados sufre un triple efecto: mientras que disminuye el trabajo remunerado, aumenta el remunerado informal y sobre todo el no remunerado ${ }^{(16)}$.

Las políticas de recuperación de las crisis dejan a las mujeres atrás. Para hacer frente a la crisis de 2008, muchos países europeos como Francia y Alemania desarrollaron planes de recuperación y políticas de mercado laboral que apoyaron sobre todo a sectores masculinizados ${ }^{(17)}$. En España, los programas de estímulo estaban vinculados principalmente a la industria de la construcción y la fabricación tradicional, de forma que más del 95\% de los puestos de trabajo creados en España mediante paquetes de estímulo estaban ocupados por hombres ${ }^{(18)}$.

La flexibilidad laboral impulsada por necesidades de las empresas, puede tener un impacto negativo sobre la salud que puede ser diferente en mujeres y hombres y según los contextos, especialmente según las tipologías de los estados de bienestar. Las medidas para disminuir las desigualdades de género en salud relacionadas con la flexibilidad laboral tienen que implicar diferentes niveles, pero atendiendo al potencial impacto, las más importantes son las que inciden en el contexto socioeconómico. Las políticas macroeconómicas, las relacionadas con el mercado de trabajo y las que conforman el estado del bienestar, así como las políticas de familia, entre otras, han de tener en cuenta el potencial efecto desigual en hombres y mujeres y se tiene que evaluar este efecto. Habrá que tener presentes las lecciones aprendidas de anteriores crisis económicas para afrontar el impacto en las desigualdades de género relacionadas con el trabajo de la actual crisis sanitaria. Podría ser una oportunidad para crear nuevos puestos de trabajo de calidad y avanzar en la igualdad de género también en este ámbito, lo que en definitiva significaría un efecto beneficioso para la salud de la población. 


\section{Bibliografía}

1. Rubery J, Piasna A. Labour market segmentation and deregulation of employment protection in the EU. En: Piasna A, Myant M, editores. Myths of Employment Deregulation: How It Neither Creates Jobs nor Reduces Labour Market Segmentation. Brussels: ETUC; 2017. p. 43-46. Disponible en: http://pinguet.free.fr/etuideregula.pdf\#page $=42$

2. Benach J, Muntaner C, Santana V. Employment Conditions and Health Inequalities. Final Report to the WHO Commission on Social Determinants of Health.; 2007. [actualizado 8 Oct 2021; citado 8 Oct 2021]. Disponible en: https://www.who. int/teams/social-determinants-of-health/equity-and-health/commission-on-social-determinants-of-health

3. Pirani E, Salvini S. Is temporary employment damaging to health? A longitudinal study on Italian workers. Soc Sci Med. 2015;124:121-131. doi: 10.1016/j.socscimed.2014.11.033

4. Campos-Serna J, Ronda-Pérez E, Artazcoz L, Moen B, Benavides F. Gender inequalities in occupational health related to the unequal distribution of working and employment conditions: a systematic review. Int J Equity Health. 2013;12(1):1-18.

5. Joyce K, Pabayo R, Critchley J, Bambra C. Flexible working conditions and their effects on employee health and wellbeing (Review). Cochrane Database Syst Rev. 2010;2.

6. Joyce K, Pabayo R, Critchley JA, Bambra C. Flexible working conditions and their effects on employee health and wellbeing. Cochrane Database Syst Rev. 2010;2010(2):CD008009. doi: 10.1002/14651858.CD008009.pub2

7. Kivimäki M, Jokela M, Nyberg S, et al. Long working hours and risk of coronary heart disease and stroke: a systematic review and meta-analysis of published and unpublished data for 603838 individuals. Lancet. 2015;386(10005):1739-1746.

8. Bannai A, Tamakoshi A. The association between long working hours and health: a systematic review of epidemiological evidence. Scand J Work Environ Health. 2014;40(1):5-18. doi: 10.5271/sjweh.3388.

9. Artazcoz L, Borrell C, Cortès I, Escribà-Agüir V, Cascant L. Occupational epidemiology and work related inequalities in health: a gender perspective for two complementary approaches to work and health research. J Epidemiol Community Health. 2007;61 Suppl 2(Suppl 2):ii39-45. doi: 10.1136/jech.2007.059774

10. Kim IH, Muntaner C, Vahid Shahidi F, Vives A, Vanroelen C, Benach J. Welfare states, flexible employment, and health: A critical review. Health Policy (New York). 2012;104:99-127. doi: 10.1016/j.healthpol.2011.11.002

11. Cortès-Franch I, Puig-Barrachina V, Vargas-Leguás H, Arcas MM, Artazcoz L. Is being employed always better for mental wellbeing than being unemployed? Exploring the role of gender and welfare state regimes during the economic crisis. Int J Environ Res Public Health. 2019;16(23):1-16. doi: 10.3390/ijerph16234799 
12. Korpi W, Ferrarini T, Englund S. Women's opportunities under different family policy constellations: Gender, class, and inequality tradeoffs in western countries re-examined. Soc Polit. 2013;20:1-40. doi: 10.1093/sp/jxs028

13. Cortès-Franch I, Escribà-Agüir V, Benach J, Artazcoz L. Employment stability and mental health in Spain: towards understanding the influence of gender and partner/marital status. BMC Public Health. 2018;18(1):425. doi: 10.1186/s12889018-5282-3

14. ILO. Policy Brief. Geneva; 2020. [actualizado 26 Jul 2003; citado 26 Sep 2003]. Disponible en: https://www.ilo.org/emppolicy/pubs/WCMS_746031/lang--en/index.htm

15. Artazcoz L, Cortès I, Benavides FG, et al. Long working hours and health in Europe: Gender and welfare state differences in a context of economic crisis. Health Place. 2016;40(June):161-168. doi: 10.1016/j.healthplace.2016.06.004

16. Cortès-Franch I. Flexibilitat laboral i salut. Aprofundint en les desigualtats de gènere i el rol dels contextos. Barcelona: Universidad Autónoma de Barcelona; 2020.

17. Gálvez L, Rodríguez P. La desigualdad de género en las crisis económicas. Investig Fem. 2012;2:113-132.

18. Eydoux A. Women during Recessions in France and Germany. Rev I'OFCE. 2014;2:153-188.

19. Gálvez-Muñoz L, Rodríguez-Modroño P. Gendered impacts of the financial, social and political crisis and austerity measures in Spain. In: The Impacts of the Crisis on Gender Equality and Women's Wellbeing in EU Mediterranean Countries. United Nations Interregional Crime and Justice Research Institute; 2013. p. 191252. Disponible en: http://www.unicri.it/sites/default/files/2019-11/Report.pdf 